\title{
La calidad del servicio al cliente en los grandes supermercados de lbagué: un análisis desde la escala multidimensional (SERVQUAL)*
}

Customer Service Quality in Large Supermarkets in Ibagué, Colombia: Analysis through the SERVQUAL Multidimensional Scale La qualité du service au client dans les grandes surfaces à Ibague : une analyse à partir de l’échelle multidimensionnelle (SERVQUAL)

\section{Resumen}

La globalización está afectando a todas las economías y a sus organizaciones que buscan maximizar sus ganancias a través de la satisfacción del cliente. Dentro de este contexto los grandes supermercados han tenido un significativo crecimiento, por lo tanto es importante conocer la calidad del servicio que los clientes están recibiendo de estas tiendas. En la actualidad las medidas de calidad de servicio en estos almacenes en el mercado mundial son escasas. Este artículo presenta los resultados de la medición de la calidad del servicio que los clientes reciben de los grandes supermercados de Ibagué, mediante el empleo de la escala multi-ítem SERVQUAL (calidad del servicio por sus siglas en inglés), que es una herramienta aplicada a la calidad del servicio, que se compone de 5 dimensiones en 22 ítems y evalúa características relacionadas con los aspectos tangibles, la confiabilidad, la responsabilidad, la garantía, la seguridad y la empatía. La población estudiada son los clientes que visitan estos supermercados con un enfoque mixto de investigación (cualitativa y cuantitativa) y los resultados mostraron la insatisfacción de los consumidores con la amabilidad del personal, el servicio de atención al cliente, la agilidad en la atención, la atención al cliente, el comportamiento del personal de seguridad, impulsadoras, mercaderistas, cajeros, empacadores y supervisores.

Palabras clave: calidad del servicio en las ventas al por menor, calidad percibida, dimensiones de la calidad y escalas de medición (SERVQUAL), grandes supermercados.

\section{Abstract}

Clasificación JEL: M30, M31

Globalization is affecting all economies and their organizations seeking to maximize their profits through customer satisfaction. Within this context, large supermarkets have had significant growth; hence, it is important to know the quality of the service customers are receiving in these stores. Currently, service quality measures in these stores in the global market are scarce. This article presents the results of the measurement of the quality of service customers receive from large supermarkets in Ibagué - Colombia, by using the SERVQUAL multi-item scale, which is a tool applied to service quality, comprising five dimensions in 22 items and evaluating characteristics related to tangible aspects, reliability, responsibility, guaranty, security, and empathy. The population studied are customers who visit these supermarkets with mixed research approach (qualitative and quantitative); the results showed customer satisfaction with personnel kindness, customer attention services, agility in attention, customer care, behavior of security personnel, product promoters, merchandisers, cashiers, packers, and supervisors.

Keywords: service quality in retail sales, perceived quality, quality dimensions and measurement Scales (SERVQUAL), large supermarkets.

\section{Résumée}

La globalisation affecte toutes les économies et les organisations qui cherchent à maximiser les revenus par le biais de la satisfaction du client. Dans ce contexte, les grandes surfaces ont eu une croissance considérable, d'où résulte important de connaître la qualité du service que les clients reçoivent dans ces établissements. Actuellement au niveau du marché mondial, ils existent quelques

\footnotetext{
* Los resultados del artículo son producto de una investigación que se realizó dentro del Grupo de Investigación en Desarrollo Económico y Empresarial de la Facultad de Ciencias Económicas y Administrativas de la Universidad del Tolima (GIDEUT).
} 
indicateurs de qualité de service dans ces magasins. Cet article présente les résultats de l'évaluation de la qualité du service que les clients reçoivent dans les grandes surfaces dans la ville d'Ibagué, à partir de l'usage de l'échelle multi-item SERVQUAL (qualité du service par ses initiales en anglais). C'est un outil appliqué à la qualité du service, composé par 5 dimensions sur 22 items, il évalue les caractéristiques en relation avec les aspects tangibles, la fiabilité, la responsabilité, la garantie, la sécurité et l'empathie. La population étudiée correspond aux clients qui visitent ces grandes surfaces ; on a utilisé une approche de recherche mixte (qualitative et quantitative). Les résultats ont montré l'insatisfaction des consommateurs face à l'amabilité du personnel, au service d' attention au client, à l'agilité de l'attention, à l'attention au client, au comportement du personnel de sécurité, aux marchandeurs, aux emballeurs, aux caissiers et aux superviseurs.

Mots clef: qualité du service dans la grande distribution, qualité perçue, dimensions de la qualité et échelles de mesure (SERVQUAL), grandes surfaces.

\section{Introducción}

La calidad del servicio se constituye en una prioridad competitiva (Miltenburg, 2009; Skinner, 1969; Hayes y Wheelwright, 1984; Martín-Peña y Díaz-Garrido, 2008) y como tal es un factor que está presente en todas las interacciones de las personas con las organizaciones y constituye un atributo diferenciador entre las empresas exitosas y las que no lo son. En la actualidad los grandes supermercados se constituyen en los sitios de mayor concentración de compradores que buscan diferentes tipos de productos y servicios. El presente artículo presenta los resultados de la investigación "El análisis de la percepción de los clientes acerca de la calidad del servicio ofrecido en los grandes supermercados de la ciudad de Ibagué", realizada por el Grupo de Investigación en Desarrollo Económico y Empresarial de la Facultad de Ciencias Económicas y Administrativas de la Universidad del Tolima, (GIDEUT), que tuvo como objetivo conocer la impresión que los consumidores tenían acerca del servicio que reciben de los supermercados Carrefour, Éxito, Mercacentro, Makro, Homecenter y Yep y a partir de allí determinar las variables más sensibles para ellos y que a la postre servirían para la toma de decisiones por parte de esas organizaciones.

En este sentido el estudio analizó la percepción de 380 clientes distribuidos de manera proporcional en estos almacenes, siguiendo la metodología de la escala SERVQUAL de Parasuraman, Zeithaml y Berry (1985), con el propósito de sistematizar las respuestas con base en las dimensiones de la calidad ofrecidas por esta herramienta y tener un diagnóstico concreto acerca de la calidad del servicio que ofrecen estos supermercados a sus clientes.

\section{Marco teórico}

Las ventas al por menor siguiendo la clasificación de Kagira y Kimani (2010), comprenden los supermercados, tiendas pequeñas, vendedores ambulantes, carnicerías, estaciones de combustible, tiendas de libros, hoteles, farmacéuticos, bancos, tiendas ordinarias, distribuidores de autos, tiendas de ropa, tiendas de joyas, tiendas de regalos, tiendas de zapatos, tiendas de muebles, tiendas de música y salones de belleza entre otros, que han tenido un crecimiento significativo y en criterio de Kimani, Kagira, Kendi y Wawire (2012), se han vuelto muy importantes actualmente en muchas economías y sobre todo en el caso de Kenia donde juegan un papel crucial en su desarrollo económico, lo cual se explica como consecuencia de la globalización, que abrió las puertas de los diferentes países para que operadores de productos y servicios extranjeros, se establecieran en determinados lugares en forma paralela con los inversores locales. Al respecto Seth, Deshmukh y Vrat (2004) exponen que:

Hoy la globalización y la liberalización están afectando no solo a las economías en desarrollo sino también a los países desarrollados. Las áreas de enfoque para las organizaciones también están cambiando de la maximización de las ganancias a maximizar los beneficios a través del incremento de la satisfacción del consumidor (p. 914).

En el caso particular del trabajo en cuestión su objeto de estudio fueron los grandes supermercados de Ibagué, que de acuerdo a Kagira y Kimani (2010), ofrecen todos los bienes y servicios señalados en su clasificación, en un ambiente caracterizado por la intensificación de la competencia, la introducción de nuevas tecnologías, sistemas de administración avanzados y la consolidación de la industria (Sirohi, McLaughlin y Witting, 1998). Según Meng, Summey, Herndon y Kwong (2009), citando a Aurifeille, Quester, Lockshin y Spawton (2002) y a Liu (2007), a propósito de las operaciones internacionales de las ventas al menudeo argumentan que:

Los minoristas de los Estados Unidos tales como Wal-Mart, 7-eleven, McDonald's y otros, derivan ingresos significativos de sus operaciones internacionales y están continuando su expansión en los mercados extranjeros. El desarrollo de estrategias efectivas de ventas al por menor que son sensibles a las diferencias transculturales parecería ser de importancia considerable en su éxito en el mercado global, especialmente cuando los minoristas locales pueden entender y responder mejor a las preferencias y condiciones locales (p. 773-774).

Estos antecedentes exigen de los grandes supermercados una nueva dinámica en la atención a sus clientes y en este sentido deben conocer sus aspectos sensibles, para lo cual se dispone de diferentes herramientas de medición, que permiten establecer su grado de satisfacción en relación con la calidad del servicio suministrado. En este sentido Siu y Chow (2003), plantean que "las medidas actuales de la calidad del servicio de los co- 
mestibles al por menor en el mercado global son escasas" (p.71), coincidiendo con Parasuraman, et al. (1985).

Los grandes supermercados han revolucionado la industria del consumo masivo, colocando a disposición de los consumidores productos y servicios en las más variadas formas, marcas, instalaciones, comodidades y precios, que indudablemente han mejorado la vida de las personas, pero no poseen políticas de medición con instrumentos sistemáticos, confiables y válidos, que les suministre información fidedigna acerca del comportamiento de sus clientes para la toma de decisiones, es decir, lo que Parasuraman, Zeithaml y Berry (1988), esquematizaron al afirmar que muchas de las medidas son desarrolladas por el capricho de un investigador que de manera subjetiva a través de un sí o un no califica las variables objeto de un estudio.

Según Crosby (1979) y Garvin (1983), a diferencia de la calidad en los bienes, los cuales pueden ser medidos objetivamente a través de indicadores como su durabilidad y número de defectos. Para Parasuraman et al. (1985), la calidad en los servicios es un concepto abstracto e indeterminado por sus características de intangibilidad, heterogeneidad e inseparabilidad de la producción y consumo. A su vez Kotler, (2000) argumenta que los investigadores de marketing, definen el término calidad como la totalidad de características de un producto o servicio, que le confieren su habilidad para satisfacer necesidades explícitas o implícitas e igualmente que la calidad puede representarse de manera objetiva y subjetiva, en el primero de los casos se refiere a la calidad actual y en el segundo caso a la percepción de la calidad del consumidor. Zeithaml (1988), puntualiza que la calidad percibida representa los juicios de los consumidores con respecto a la excelencia o superioridad de las marcas globales.

Parasuraman, Zeithaml y Berry (1994), enfatizan que "nuestra investigación provee un fuerte apoyo para la definición de calidad del servicio como la discrepancia entre las expectativas y percepciones de los consumidores" (p.111). Radomir, Plaias y Nistor (2012), a propósito de la conceptualización acerca de la calidad del servicio, subrayan los aspectos principales que necesitan ser tomados en cuenta cuando se intenta explicar el concepto de calidad del servicio, siendo ellos los relacionados con las características que diferencian los productos de los servicios, coincidiendo con Parasuraman et al. (1985) y que por lo tanto pueden ser considerados "una causa" de la ambigüedad asociada con el término de calidad del servicio, en la misma dirección de Garvin (1988: xi), citado por Holbrook (1994), cuando expone que la "calidad es un concepto extraordinariamente resbaladizo, fácil de visualizar pero exasperantemente difícil de definir" (p.407).

Igualmente Radomir et al. (2012), abordan diferentes conceptos de este término, iniciando con Crosby (1979) y su argumento acerca de la "conformidad con los requerimientos", hasta Santhiyavalli y Sandhya (2011), quienes plantean que "la calidad del servicio es una función de la diferencia en resultados o brechas entre la expectativas y las percepciones", pasando por Olshavsky (1985), quien expone que la calidad es una forma de evaluación total de un producto, similar en muchas maneras a la actitud; Juran (1988), la califica como la "aptitud para el uso"; Lewis (1989), quien expone que "la calidad del servicio percibida es un juicio del consumidor (una forma de actitud) y los resultados de las comparaciones que los clientes hacen entre sus expectativas y sus percepciones del desempeño actual del servicio y Boulding, Kalra, Staelin y Zeithaml (1993), quienes argumentan que la

“...percepción actual de los individuos acerca de la calidad del servicio de una empresa justo después del contacto son una mezcla de 1) sus expectativas anteriores de que será y lo que debe transcurrir durante el contacto y 2) el servicio de entrega actual durante el encuentro del servicio".

Por otra parte Kimani et al. (2012), con respecto a la percepción del cliente y citando a Bitner y Hubbert (1994), la define como la impresión total del consumidor acerca de la inferioridad o superioridad relativa de una organización y sus servicios; esta impresión es influida por muchos factores tales como el desempeño de los empleados, instalaciones, precios de los productos y calidad del servicio ofrecido entre otros aspectos. Un criterio subyacente y de suma importancia que destacan Kimani et al. (2012), dentro del proceso de valoración de la calidad del servicio ofrecido a los clientes por los almacenes de ventas al por menor, son los instrumentos de medición sobre los cuales resaltan en especial la escala SERVQUAL (Calidad del Servicio por su siglas en inglés), desarrollada por Parasuraman et al. $(1985,1988)$ y RSQS (Escala de Calidad de los Servicios en las ventas al por menor), elaborada por Dabholkar, Thorpe y Tentz (1996). Además de estos métodos, Meng et al. (2009) presentan el modelo de percepción del servicio (SERVPERF) elaborada por Cronin y Taylor (1992).

\section{Dimensiones de la calidad del servicio en los grandes supermercados de lbagué}

Como se expuso antes para efectos de la medición de las dimensiones e ítems de la calidad del servicio en los grandes supermercados de lbagué, se tomó como referencia la escala multi-item SERVQUAL de Parasuraman et al. $(1985,1988)$, la cual ha sido ampliamente conocida, discutida, aplicada y validada en diferentes industrias de servicio, tales como hospitales, educación y turismo, pero que aún no ha sido adoptada exitosamente en el ambiente de las tiendas de ventas al por menor (Huang, 2009). Este estudio propone inicialmente las siguientes hipótesis:

H1: La calidad del servicio en los grandes supermercados de Ibagué tiene un impacto positivo sobre la calidad percibida de los clientes. 
Aspectos tangibles: en esta dimensión se incluyeron todas aquellas variables relacionadas con las instalaciones físicas como el parqueadero, amplitud de los pasillos, orientación y señalización, calidad de los productos, surtido y facilidades de pago.

H2: Los aspectos tangibles tienen un impacto positivo sobre el nivel de satisfacción del servicio recibido en los grandes supermercados de lbagué.

Confiabilidad: se incluyeron en esta dimensión los ítems de garantía y servicio posventa y claridad en la información.

H3: La confiabilidad tiene un impacto positivo sobre el nivel de satisfacción del servicio recibido en los grandes supermercados de lbagué.

Responsabilidad: se localizaron aquí la atención profesional, la claridad en la información de la oficina de servicio al cliente, interés por la situación de los clientes, rapidez en la respuesta y efectividad en la solución de los problemas.

H4: La responsabilidad tiene un impacto positivo sobre el nivel de satisfacción del servicio recibido en los grandes supermercados de Ibagué.

Seguridad: en esta dimensión quedaron incluidos los ítems amabilidad del personal, atención al cliente, agilidad en la atención, vigilantes, impulsadoras-mercaderistas, cajeros, empacadores y supervisores

H5: La seguridad tiene un impacto positivo sobre el nivel de satisfacción del servicio recibido en los grandes supermercados de Ibagué.

Empatía: fueron parte de esta dimensión la cercanía de la superficie y el horario de atención.

H6: La empatía tiene un impacto positivo sobre el nivel de satisfacción del servicio recibido en los grandes supermercados de Ibagué.

\section{Metodología}

El estudio utilizó un enfoque mixto de investigación, es decir, abordó aspectos relacionados con la investigación cuantitativa en tanto "que busca medir fenómenos sociales: ella ofrece una expresión cifrada a los datos y los analiza con la ayuda de métodos estadísticos" (Deslauries, 2004, p.19). Igualmente fue cualitativa en la medida que "puede ofrecer una visión más holística y más global de la realidad social: está inmersa en el tiempo real de las personas, no en el tiem- po experimental del laboratorio" (Deslauries, 2004, p.22). Gómez, Deslauries y Alzate (2010) al respecto exponen que "hoy es posible encontrar y concebir, como se ha planteado, metodologías mixtas donde los datos cualitativos están emparentados con los datos cuantitativos con el fin de enriquecer la metodología $y$, eventualmente, los resultados de la investigación" (p.101).

Asimismo esta investigación se enmarcó en los enfoques descriptivo y explicativo, en el primero de ellos "se ocupa de la descripción de las características que identifican los diferentes elementos o componentes y su interrelación" (Méndez, 1995, p.125), "el problema está estructurado y bien entendido" (Ghauri y Gronhaug, 2010, p.56); es explicativo porque identifica y analiza las causales (variables independientes) y sus resultados, los que se expresan en hechos verificables (variables dependientes) (Méndez, 1995, p.127) y transversal porque se recolectan datos en un solo momento, en un tiempo único (Hernández, Fernández y Baptista, 2010, p.151).

La recolección de los datos se realizó a través de un cuestionario estructurado, aplicado en cada una de las 6 tiendas de manera proporcional, en el cual los ítems fueron sistematizados de acuerdo con la escala SERVQUAL y asignados a cada una de sus dimensiones: tangibles, garantía, seguridad, responsabilidad, confiabilidad y empatía. La selección de los encuestados se realizó a través de un muestreo sistemático lineal (MSL) (Ospina, 2001; Lind, Marchal y Mason, 2004), a los visitantes de estos supermercados para un total de 380 instrumentos aplicados. El MSL fue empleado en razón a que no se disponía de un marco muestral específico, del cual se pudiera extraer una muestra de manera aleatoria por tratarse de una población dispersa. La encuesta fue contestada por 213 hombres y 167 mujeres. Se trató de un cuestionario estructurado de 13 preguntas con 50 variables, que pretendían medir de forma general el grado de satisfacción de los compradores de estos supermercados, pero que para efectos de este estudio se seleccionaron aquellos ítems que presentaban mayor relación con la escala SERVQUAL modificada por Parasuraman, Zeithaml y Berry (1991), quedando al final solo 25 items que fueron calificados a través de una escala tipo Likert.

El proceso estadístico fue realizado con SPSS 21 y comprendió un análisis factorial por componentes principales con rotación varimax, con el propósito de reducir un conjunto relativamente extenso de variables observables a un número reducido de variables hipotéticas llamadas factores (Martín, Cabero y De Paz, 2008) e igualmente según Hair, Anderson, Tatham y Black (1999) para "analizar interrelaciones entre un gran número de variables y explicar estas variables en términos de sus dimensiones subyacentes comunes (factores)" (p.11). 


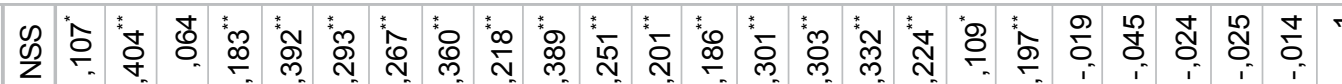

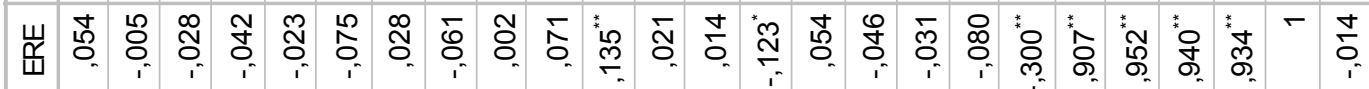

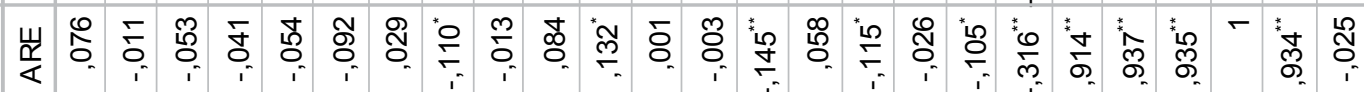

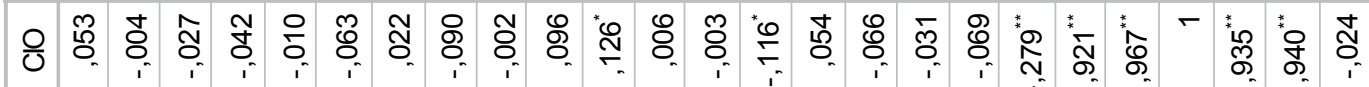

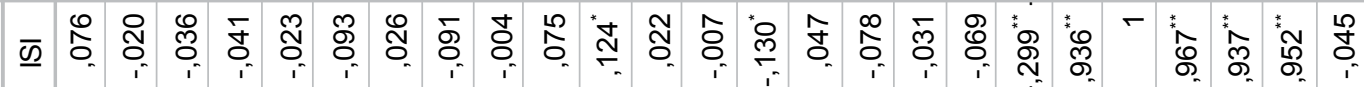

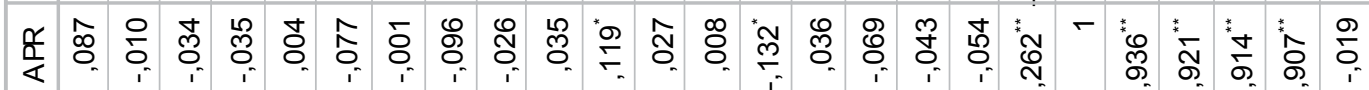

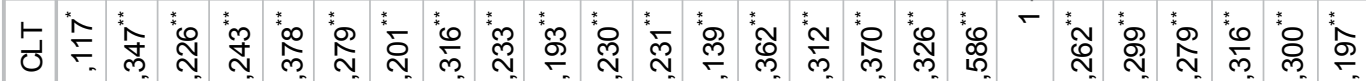

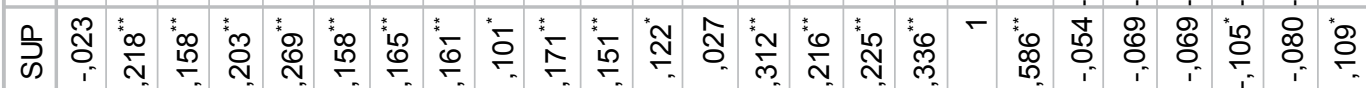

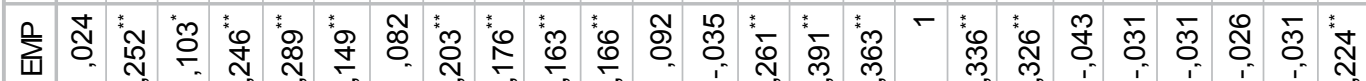

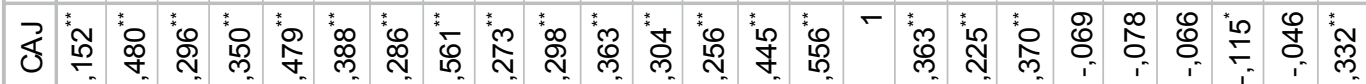

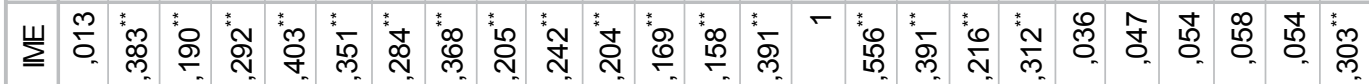

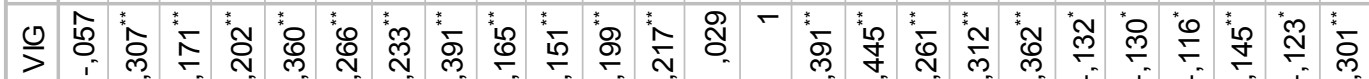

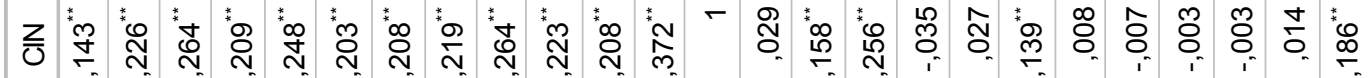

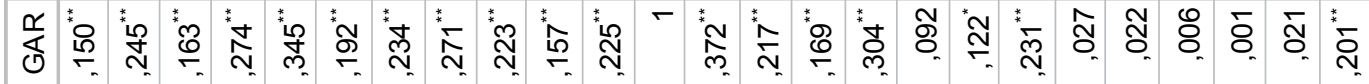

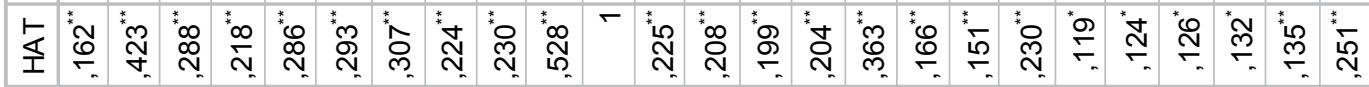

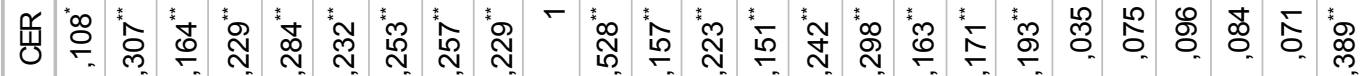

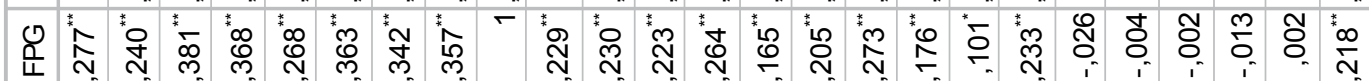

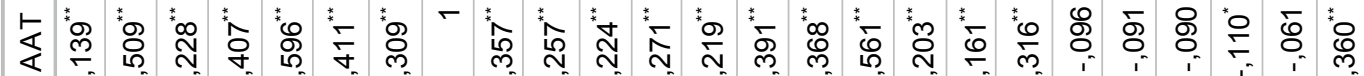

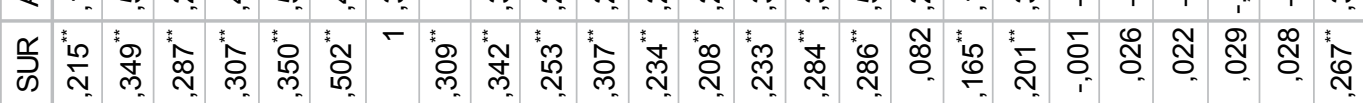

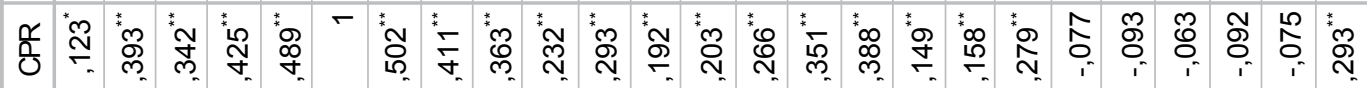

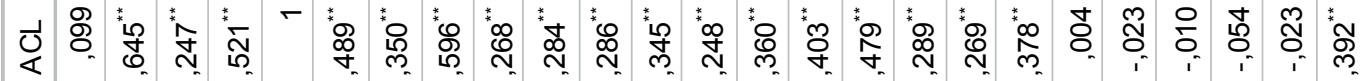

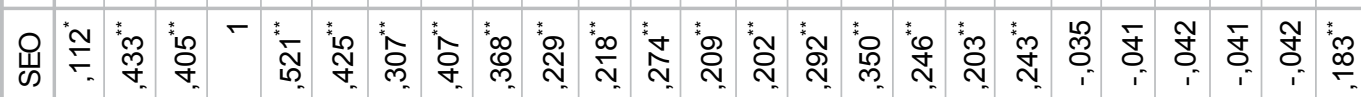

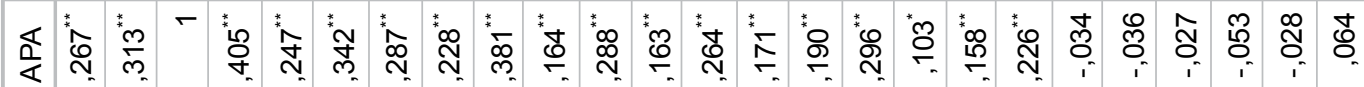

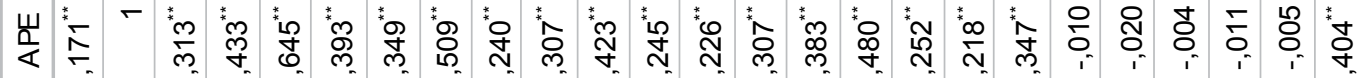

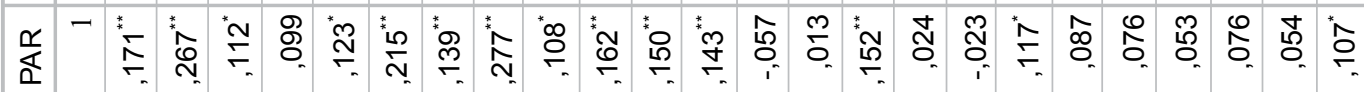

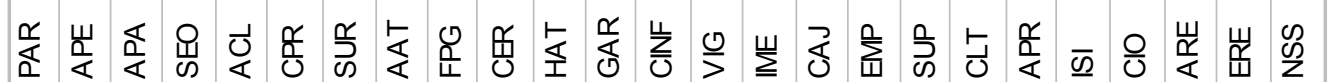


Adicionalmente fue usado el coeficiente de correlación de Pearson para determinar, según Guisande, Vaamonde y Barreiro (2011) "si existe asociación entre dos o más caracteres o variables de una misma muestra. Además de conocer si dos o más variables son independientes o dependientes, es interesante conocer también, cuál es la fuerza de dicha asociación" (p.189), con lo cual se confirma también la validez de constructo (convergente y discriminante). En este mismo sentido Kimani et al. (2012), plantearon que el coeficiente de correlación de Pearson se usa para determinar la naturaleza de las relaciones entre variables. La validez de contenido se soporta en la respectiva revisión de literatura de investigaciones realizadas sobre las características de la calidad del servicio, calidad percibida y las escalas de medición para estas dimensiones que se reseñaron en el apartado correspondiente, así como una muestra piloto a 47 clientes que permitió ajustar el instrumento de recolección de datos. El indicador de confiabilidad se realizó a través de la técnica de análisis factorial por componentes principales y del cálculo del coeficiente alfa de Cronbach tanto para los ítems de cada dimensión como para todos los ítems del cuestionario. Por último con los diferentes procedimientos estadísticos que se han descrito se pretenden contrastar las hipótesis del estudio.

\section{Fiabilidad y validez de las escalas de medición}

Con el propósito de medir el instrumento de recolección de datos desde las perspectivas del grado de confiabilidad y validez, se utilizó el coeficiente alfa de Cronbach (Cronbach, 1951), el cual arrojó un índice de 0,81 para los ítems del cuestionario y para las dimensiones de la calidad del servicio de la escala SERVQUAL de Parasuraman et al. (1991), este indicador se presentó de la siguiente forma: tangibles 0,63 ; confiabilidad 0,73 ; responsabilidad 0,61; seguridad 0,71; y empatía 0,97. Como se observa en todos los casos, el estadístico Alpha de Cronbach arrojó valores superiores a 0,6 que de acuerdo con Hair, et al. (1999), es el valor mínimo requerido para un análisis exploratorio e incluso algunos de ellos superiores a 0,7 que según Nunally (1978), es el recomendado para análisis confirmatorios; también quedó demostrada la consistencia interna entre las variables que conformaron el instrumento.

El análisis factorial exploratorio se inicia con el cálculo de las correlaciones entre las variables cuyos resultados se muestran en la Tabla 1, allí se puede evidenciar que existen relaciones significativas con el $1 \%$ y $5 \%$, siendo su determinante igual a 1,249E-007 que es un valor muy bajo que indica que las variables están bastante correlacionadas (Martín et al. 2008), lo cual corrobora de hecho la existencia de validez convergente. Además, la prueba de esfericidad de Bartlett (Tabla 2), con un estadístico de contraste de 5862,751 , con 300 grados de libertad y significación de 0 , permite concluir que significativamente a cualquier nivel existe correlación entre algunas variables. En esta misma tabla aparece la medida de adecuación de la muestra que es el KOM (índice de Kaiser, Meyer y Oklin), según el baremo de Kaiser este estadístico de o,864 se considera excelente (Guisande et al. 2011) y significa que las correlaciones entre pares de variables pueden ser explicadas por las otras variables (Martín et al. 2008).

\begin{tabular}{|c|c|c|}
\hline \multicolumn{3}{|c|}{ Tabla 2. KMO y prueba de Bartlett } \\
\hline \multicolumn{2}{|c|}{ Medida de adecuación muestral de Kaiser-Meyer-Olkin } & ,864 \\
\hline \multirow{3}{*}{$\begin{array}{l}\text { Prueba de esfericidad de } \\
\text { Bartlett }\end{array}$} & Chi-cuadrado aproximado & 5862,751 \\
\hline & gl & 300 \\
\hline & Sig. & 0,000 \\
\hline & Fuente: Rubio (2014) a part & de datos. \\
\hline
\end{tabular}

Una vez establecido el grado de correlación de las variables objeto de análisis, corresponde ahora encontrar la varianza total explicada y extraer el número de factores con autovalores mayores que 1 siguiendo el criterio de Kaiser para un total de 6, mediante el método de componentes principales con rotación varimax que explican la varianza total en un $62,2 \%$ y que representan a las 25 variables originales. Se trata en este caso de que en cada factor queden variables muy parecidas entre ellas pero muy diferentes a las que se ubican en otros factores (Tabla 3).

En cuanto al análisis factorial este se realizó a partir de las 25 variables incluidas en el cuestionario, empleando la técnica de componentes principales con rotación varimax; se estableció como criterio de saturación que los "eigen valores" o valores propios fueran iguales o superiores a uno y un factor de carga igual a 0,40 (Tabla 4).

En la matriz de componentes rotados se pueden observar 6 factores que agruparon los 25 ítems estudiados: del primer factor denominado "responsabilidad" hacen parte las variables profesionalismo en la atención, interés en la situación de los clientes, claridad en la información de la oficina de servicio al cliente, rapidez en la respuesta a los requerimientos de los consumidores y efectividad en la solución de los problemas de los clientes. El segundo factor "seguridad" comprende las variables amabilidad del personal, atención al cliente, agilidad en la atención, personal de seguridad, impulsadoras y mercaderistas, cajeros, empacadores y supervisores; el tercer factor "tangibles" quedó constituido por accesibilidad al parqueadero, amplitud de los pasillos, orientación-señalización, calidad de los productos, surtido y facilidades de pago. El cuarto factor "confiabilidad" agrupó garantía y servicio posventa y claridad en la información. El quinto factor "empatía" lo conformaron las variables ubicación del supermercado y el horario de atención y por último el sexto factor que podría etiquetarse como "calidad" y se consolidó con los ítems calidad del servicio y calidad percibida. Como se deduce de esta matriz, los ítems de las dimensiones iniciales de SERVQUAL se reclasificaron con base en el análisis factorial. 


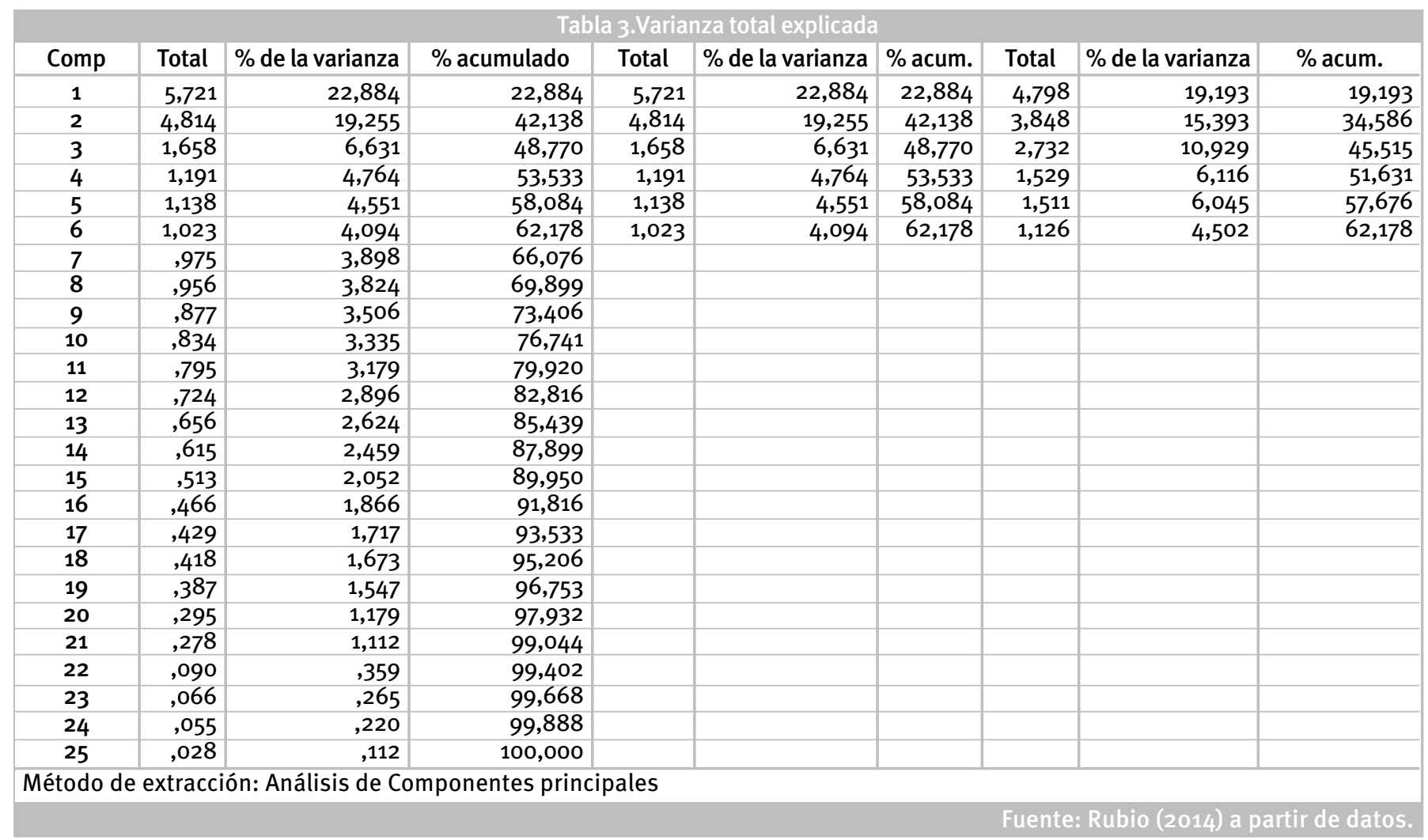

\begin{tabular}{|c|c|c|c|c|c|c|}
\hline \multicolumn{7}{|c|}{ Tabla 4. Matriz de componentes rotadosa } \\
\hline & \multicolumn{6}{|c|}{ Componente } \\
\hline & 1 & 2 & 3 & 4 & 5 & 6 \\
\hline ACCE.PARQ & , 073 &,- 155 &, 577 & 122 & 085 & ,163 \\
\hline AMAB.PERS & ,011 &, 571 &, 349 & ,182 & ,203 &,- 022 \\
\hline AMP.PASILL &,- 042 & ,127 &, 664 &, 063 & ,116 & ,138 \\
\hline ORIENT.SEÑ &,- 020 & ,442 &, 535 & ,110 &,- 059 &,- 068 \\
\hline ATEN.CLTE & ,008 & ,682 &, 328 & ,248 & ,012 &,- 091 \\
\hline CAL.PROD &,- 074 & ,406 &, 579 & ,038 & ,063 &,- 092 \\
\hline SURTIDO &, 028 & ,249 &, 578 &, 024 & ,198 &,- 192 \\
\hline AGIL.ATENC &,- 073 & ,609 &, 302 &, 317 &,- 023 &,- 064 \\
\hline FAC.PAGO &,- 007 & ,154 & ,654 & ,119 & ,076 &,- 066 \\
\hline UBIC.SUP & ,057 & ,210 & ,156 &, 080 & ,799 &,- 070 \\
\hline HOR.ATEN & ,119 & ,215 &, 256 & 096 &, 771 & ,072 \\
\hline GAR.S.POS &, 029 &, 265 & ,130 &, 663 & ,047 &,- 039 \\
\hline CLAR.INF & ,002 &, 042 & ,272 & 677 & ,131 & ,096 \\
\hline PERS.SEG &,- 129 & ,665 &,- 035 & ,037 & ,104 &,- 209 \\
\hline IMP.MERC & ,086 & ,707 & ,094 &, 064 &, 063 & ,079 \\
\hline CAJEROS &,- 057 & 686 & ,196 & ,254 & ,173 &, 051 \\
\hline EMPACAD &,- 008 & ,608 &,- 005 &,- 233 & ,105 & ,237 \\
\hline SUPERV &,- 075 & ,496 &, 056 &,- 290 & , 180 & , 130 \\
\hline PROF.AT & ,960 &,- 029 &,- 002 & ,017 & ,004 & ,010 \\
\hline INT.SIT & ,982 &,- 034 &,- 004 & ,001 &, 036 &,- 006 \\
\hline CL.IN.OF.C. & ,975 &,- 022 &,- 007 &,- 002 &, 052 &,- 022 \\
\hline RAP.RESP & ,968 &,- 057 &,- 001 &,- 013 &, 052 &,- 006 \\
\hline EFEC.SOL & 970 &,- 020 &,- 004 &, 020 & ,035 &,- 006 \\
\hline CALSER & ,068 & ,057 & ,116 &,- 331 &,- 181 &, 587 \\
\hline CALPER &,- 085 & ,006 &,- 094 & ,233 & ,120 & ,721 \\
\hline
\end{tabular}

Método de extracción: Análisis de componentes principales. Método de rotación: Normalización varimax con Kaiser.

a. La rotación ha convergido en 7 iteraciones.

\section{Resultados y discusión}

\section{Contraste de hipótesis}

Se procede a contrastar el sistema de hipótesis planteado en la revisión de la literatura a través del criterio del coeficiente de correlación de Pearson, siguiendo la metodología usada por Kimani et al. (2012), a propósito de su estudio sobre la "percepción de los compradores acerca de la calidad del servicio de las ventas al por menor: supermercados vs. pequeñas tiendas de conveniencia (Dukas) en Kenya.

H1: La correlación lineal de Pearson en este caso es significativa al $5 \%$ con un índice de correlación de 11,5 , por lo cual se concluye que la calidad del servicio tiene una influencia relativa sobre la calidad percibida de los clientes de los grandes supermercados de Ibagué (Tabla 5).

\begin{tabular}{|l|l|r|r|}
\hline & \multicolumn{3}{|c|}{ Tabla 5. Correlaciones Calidad del servicio vs. calidad percibida } \\
\hline CALSERV & Correlación de Pearson & $\mathbf{1}$ & $\mathbf{1 1 5}$ \\
\hline CALPERC & Correlación de Pearson & $\mathbf{1 1 5}$ & $\mathbf{1}$ \\
\hline * La correlación es significante al nivel 0,05 (bilateral). \\
\hline \multicolumn{4}{|c|}{ Fuente: Rubio (2014) a partir de datos. } \\
\hline
\end{tabular}

H2: Como puede apreciarse en la Tabla 6, existe correlación significativa al $1 \%$ y $5 \%$ entre los ítems parqueadero, calidad de los productos y surtido con el nivel de satisfacción del servicio, por lo cual se puede deducir 
que existe influencia de estas variables sobre el nivel de satisfacción de los clientes de estos supermercados en cuanto a la dimensión "tangibles", con lo cual esta hipótesis se cumple parcialmente.

\begin{tabular}{|c|c|c|c|c|c|c|c|c|}
\hline & & Parq & Amp.pas & Señ/or & Cal. pro & Surt. & Fal.pag & N.sat.serv. \\
\hline Parq. & Corr.de Pearson & 1 &, $297^{\star \star}$ &, $266^{\star \star}$ &, $242^{\star \star}$ &, $239^{\star \star}$ &, $321^{\star \star}$ &, $111^{\star}$ \\
\hline Amp.pas & Corr.de Pearson &, $297^{\star \star}$ & 1 &, $599^{\star \star}$ &, $421^{\star \star}$ &, $454^{\star \star}$ &, $444^{\star \star}$ &, 092 \\
\hline Señ/Or & Corr. de Pearson &, $266^{\star \star}$ &, $599^{\star \star}$ & 1 &, $478^{\star \star}$ &, $379^{\star \star}$ &, $423^{\star \star}$ & ,092 \\
\hline Cal-pro & Corr.de Pearson &, $242^{\star \star}$ & $421^{\star \star}$ & $478^{\star \star}$ & 1 &, $614^{\star \star}$ &, $351^{\star \star}$ &, $170^{\star \star}$ \\
\hline Surt. & Corr.de Pearson &, $239^{\star \star}$ &, $454^{\star \star}$ &, $379^{\star \star}$ &, $614^{\star \star}$ & 1 &, $402^{\star \star}$ &, $209^{\star \star}$ \\
\hline Fal-pago & Corr.de Pearson &, $321^{\star \star}$ &, $444^{\star \star}$ &, $423^{\star \star}$ &, $351^{\star \star}$ &, $402^{\star \star}$ & 1 & ,084 \\
\hline N.sat.ser & Corr. De Pearson &, $111^{*}$ & ,092 & ,092 &, $170^{\star \star}$ &, $209^{\star \star}$ & ,084 & 1 \\
\hline
\end{tabular}

** La correlación es significativa al nivel 0,01 (bilateral).

* La correlación es significante al nivel o,05 (bilateral).

Fuente: Rubio (2014) a partir de datos.

H3: Como se evidencia en la Tabla 7, no existe correlación significativa entre los ítems garantía-servicio postventa y claridad en la información con relación al nivel de satisfacción del servicio, por lo cual se puede deducir que no existe influencia de estas variables sobre el nivel de satisfacción de los clientes de estos supermercados en cuanto a la dimensión de "confiabilidad”, por lo cual se rechaza esta hipótesis.

\begin{tabular}{|l|l|r|r|r|}
\hline \multicolumn{5}{|c|}{ Tabla 7. Correlaciones dimensión confiabilidad vs. nivel } \\
Satisfacción servicio \\
\hline Ni.sat-ser & Correlación de Pearson & $\begin{array}{c}\text { Ni. sat. } \\
\text { ser }\end{array}$ & $\begin{array}{c}\text { Gtía. ser. } \\
\text { post }\end{array}$ & Clar.inf \\
\hline Gtía.ser.post & Correlación de Pearson &, 060 &, 060 &,- 024 \\
\hline Clar.inf & Correlación de Pearson &,- 024 &,- 030 &,- 030 \\
\hline & Fuente: Rubio & 1 \\
\hline
\end{tabular}

H4: Como puede apreciarse en la Tabla 8, existe correlación significativa al $1 \%$ entre el ítem claridad de la información de la oficina de servicio al cliente en relación con el nivel de satisfacción del servicio, por lo cual se puede deducir que sólo esta variable tiene influencia sobre el nivel de satisfacción de los clientes de estos supermercados en cuanto a la dimensión de "responsabilidad", por lo cual esta hipótesis de cumple parcialmente.

\begin{tabular}{|c|c|c|c|c|c|c|}
\hline & $\begin{array}{l}\text { Niv.sat. } \\
\text { serv. }\end{array}$ & At.prof. & Int.sit. & Clar.inf. & $\begin{array}{c}\text { Agil.res } \\
\text { p. }\end{array}$ & $\begin{array}{l}\text { Efect.re } \\
\text { sp. }\end{array}$ \\
\hline $\begin{array}{l}\text { Nivel } \\
\text { satisfacción } \\
\text { servicio }\end{array}$ & 1 &,- 019 &,- 045 &, $186^{\star \star}$ &,- 025 &,- 014 \\
\hline $\begin{array}{l}\text { Atención } \\
\text { profesional }\end{array}$ &,- 019 & 1 &, $936^{\star \star}$ & ,008 &, $914^{\star \star}$ &, $907^{\star \star}$ \\
\hline $\begin{array}{l}\text { Interés } \\
\text { en la situación }\end{array}$ &,- 045 & ,936** & 1 &,- 007 &, $937^{\star \star}$ &, $952^{\star \star}$ \\
\hline $\begin{array}{l}\text { Claridad } \\
\text { información }\end{array}$ &, $186^{\star \star}$ & ,008 &,- 007 & 1 &,- 003 & ,014 \\
\hline $\begin{array}{l}\text { Agilidad } \\
\text { atención }\end{array}$ &,- 025 &, $914^{\star \star}$ & $937^{\star \star}$ &,- 003 & 1 &, $934^{\star \star}$ \\
\hline $\begin{array}{l}\text { Efectividad } \\
\text { respuesta }\end{array}$ &,- 014 &, $907^{\star \star}$ &, $952^{\star \star}$ & ,014 & $934^{\star \star}$ & 1 \\
\hline \multicolumn{7}{|c|}{ ** La correlación es significativa al nivel o,01 (bilateral). } \\
\hline & & Fuente & Rubio & & & e datos. \\
\hline
\end{tabular}

H5: Como puede apreciarse en la Tabla 9, existen correlaciones significativas al $1 \%$ y $5 \%$ entre todos los ítems de esta dimensión en relación con el nivel de satisfacción del servicio, por lo cual puede deducirse que todas las variables tienen influencia sobre el nivel de satisfacción de los clientes de estos supermercados en cuanto a la dimensión de "seguridad", por lo cual esta hipótesis es aceptada.

H6: Como puede apreciarse en la Tabla 10 existen correlaciones significativas al $1 \%$ entre todos los ítems de esta dimensión en relación con el nivel de satisfacción del servicio, por lo cual se puede deducir que todas las variables tienen influencia sobre el nivel de satisfacción de los clientes de estos supermercados en cuanto a la dimensión de "empatía", por lo cual esta hipótesis es aceptada.

A nivel descriptivo es importante destacar la insatisfacción de los clientes de los grandes supermercados de Ibagué en relación con las variables objeto de análisis según se observa en la Tabla 11, de allí se deducen evaluaciones regulares y deficientes con respecto a los ítems agilidad en la atención (28\%), atención al cliente $(16 \%)$, garantía y servicio postventa $(15 \%)$, precios (15\%), amabilidad del personal (13\%), claridad en la información (12,5\%), amplitud de los pasillos (12\%) y orientación y señalización (11,9\%). Del total de las variables consideradas importantes y muy importantes por los clientes de estos almacenes, $56,3 \%$ de estas no son bien calificadas por ellos, pero que en una buena proporción están correlacionadas significativamente (Tablas 1 y 11).

\section{Conclusiones}

La calidad del servicio y la satisfacción de los consumidores con respecto a los proveedores de productos y servicios, es un tema de gran importancia no sólo desde la perspectiva del cliente, sino como información básica para la toma de decisiones por parte de la gerencia de las grandes superficies de Ibagué. 
Tabla 9. Correlaciones dimensión seguridad vs. nivel de satisfacción del servicio

\begin{tabular}{|c|c|c|c|c|c|c|c|c|c|c|}
\hline & & $\begin{array}{l}\text { Niv. sat. } \\
\text { servicio }\end{array}$ & $\begin{array}{c}\text { Amp. } \\
\text { pasillos }\end{array}$ & $\begin{array}{l}\text { Atenc. } \\
\text { cliente }\end{array}$ & $\begin{array}{c}\text { Agil. } \\
\text { Atenc. }\end{array}$ & Vigil. & Im.merc & Caj. & Emp. & Sup. \\
\hline Nivel Satisfacción servicio & Corr. Pearson & 1 &, $404^{\star \star}$ &, $392^{\star \star}$ &, $360^{\star \star}$ &, $301^{\star \star}$ &, $303^{\star \star}$ &, $332^{\star \star}$ &, $224^{\star \star}$ &, $109^{*}$ \\
\hline Amplitud pasillos & Corr. Pearson &, $404^{\star \star}$ & 1 &, $645^{\star \star}$ &, $509^{\star \star}$ & $307^{\star \star}$ &, $383^{\star \star}$ &, $480^{\star \star}$ &, $252^{\star \star}$ &, $218^{\star \star}$ \\
\hline Atención al cliente & Corr. Pearson &, $392^{\star \star}$ &, $645^{\star \star}$ & 1 &, $596^{\star \star}$ & $360^{\star \star}$ &, $403^{\star \star}$ &, $479^{\star \star}$ &, $289^{\star \star}$ &, $269^{\star \star}$ \\
\hline Agilidad en la atención & Corr. Pearson &, $360^{\star *}$ &, $509^{\star \star}$ &, $596^{\star \star}$ & 1 &, $391^{\star \star}$ &, $368^{\star *}$ &, $561^{\star \star}$ & $203^{\star \star}$ &, $161^{\star \star}$ \\
\hline Vigilancia & Corr. Pearson &, $301^{\star \star}$ &, $307^{\star \star}$ &, $360 * \star$ &, $391^{\star \star}$ & 1 &, $391^{\star \star}$ & $445^{\star \star}$ &, $261^{\star \star}$ &, $312^{\star \star}$ \\
\hline Impulsadoras mercaderistas & Corr. Pearson &, $303^{\star \star}$ &, $383^{\star \star}$ & $403^{\star \star}$ &, $368^{\star \star}$ &, $391^{\star \star}$ & 1 &, $556^{\star \star}$ & $391^{\star \star}$ &, $216^{\star \star}$ \\
\hline Cajeras & Corr. Pearson &, $332^{\star \star}$ &, $480^{\star \star}$ &, $479^{\star \star}$ &, $561^{\star \star}$ &, $445^{\star \star}$ &, $556^{\star \star}$ & 1 &, $363^{\star *}$ &, $225^{\star \star}$ \\
\hline Empacadores & Corr. Pearson &, $224^{\star \star}$ &, $252^{\star \star}$ &, $289^{\star \star}$ & $203^{\star \star}$ &, $261^{\star \star}$ &, $391^{\star \star}$ &, $363^{\star \star}$ & 1 & $336^{\star \star}$ \\
\hline Supervisores & Corr. Pearson & ,109* &, $218^{\star \star}$ &, $269^{\star \star}$ &, $161^{\star \star}$ &, $312^{\star \star}$ &, $216^{\star \star}$ &, $225^{\star \star}$ & $336^{\star \star}$ & 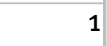 \\
\hline
\end{tabular}

**. La correlación es significativa al nivel 0,01 (bilateral).

*. La correlación es significante al nivel 0,05 (bilateral).

Fuente: Rubio (2014) a partir de datos.

Tabla 10. Correlaciones dimensión empatía vs. nive satisfacción servicio recibido

\begin{tabular}{|c|c|c|c|c|}
\hline & & $\begin{array}{c}\text { Nivel } \\
\text { sat.servicio }\end{array}$ & Cercanía & $\begin{array}{l}\text { Horario } \\
\text { atención }\end{array}$ \\
\hline $\begin{array}{l}\text { Nivel satisfacción } \\
\text { servicio }\end{array}$ & $\begin{array}{l}\text { Correlación } \\
\text { de Pearson }\end{array}$ & 1 & $389^{\star \star}$ &, $251^{\star \star}$ \\
\hline Cercanía & $\begin{array}{l}\text { Correlación } \\
\text { de Pearson }\end{array}$ &, $389^{\star \star}$ & 1 &, $528^{\star \star}$ \\
\hline Horario atención & $\begin{array}{l}\text { Correlación } \\
\text { de Pearson }\end{array}$ &, $251^{\star \star}$ &, $528 \star \star$ & 1 \\
\hline \multicolumn{5}{|c|}{ ^*. La correlación es significativa al nivel o,01 (bilateral). } \\
\hline \multicolumn{5}{|c|}{ Fuente: Rubio (2014) a partir de datos. } \\
\hline
\end{tabular}

Los resultados del estudio tienen importantes implicaciones para estas tiendas pues les permiten redireccionar sus estrategias competitivas, mediante la evaluación de sus actividades y a partir de ese proceso iniciar el mejoramiento donde sea requerido. No pueden descuidarse los factores críticos que constituyen el nivel de satisfacción del servicio recibido, por parte de los consumidores de estos supermercados y que se pusieron de presente en los resultados de la investigación en especial los criterios que han sido validados entre otros por autores como Parasuraman, et al. (1985); Siu y Chow (2003); Kagira y Kimani (2010).

En este sentido se destacan los aspectos de cada una de las dimensiones estudiadas, que se han considerado importantes desde el punto de vista de los clientes y que fueron determinados a través del análisis factorial y el coeficiente de correlación de Pearson. Los clientes conceden especial importancia al servicio del parqueadero, a la calidad de los productos y al surtido desde la dimensión de los tangibles; desde el factor de seguridad destacan la amabilidad del personal, el servicio de atención al cliente, la agilidad en la atención, la atención al cliente, el comportamiento del personal de seguridad, impulsadoras, mercaderistas, cajeros, empacadores y supervisores. También son fundamentales para los consumidores los ítems relacionados con la claridad de la información de la oficina de servicio al cliente del factor de responsabilidad. En lo relacionado con la dimensión

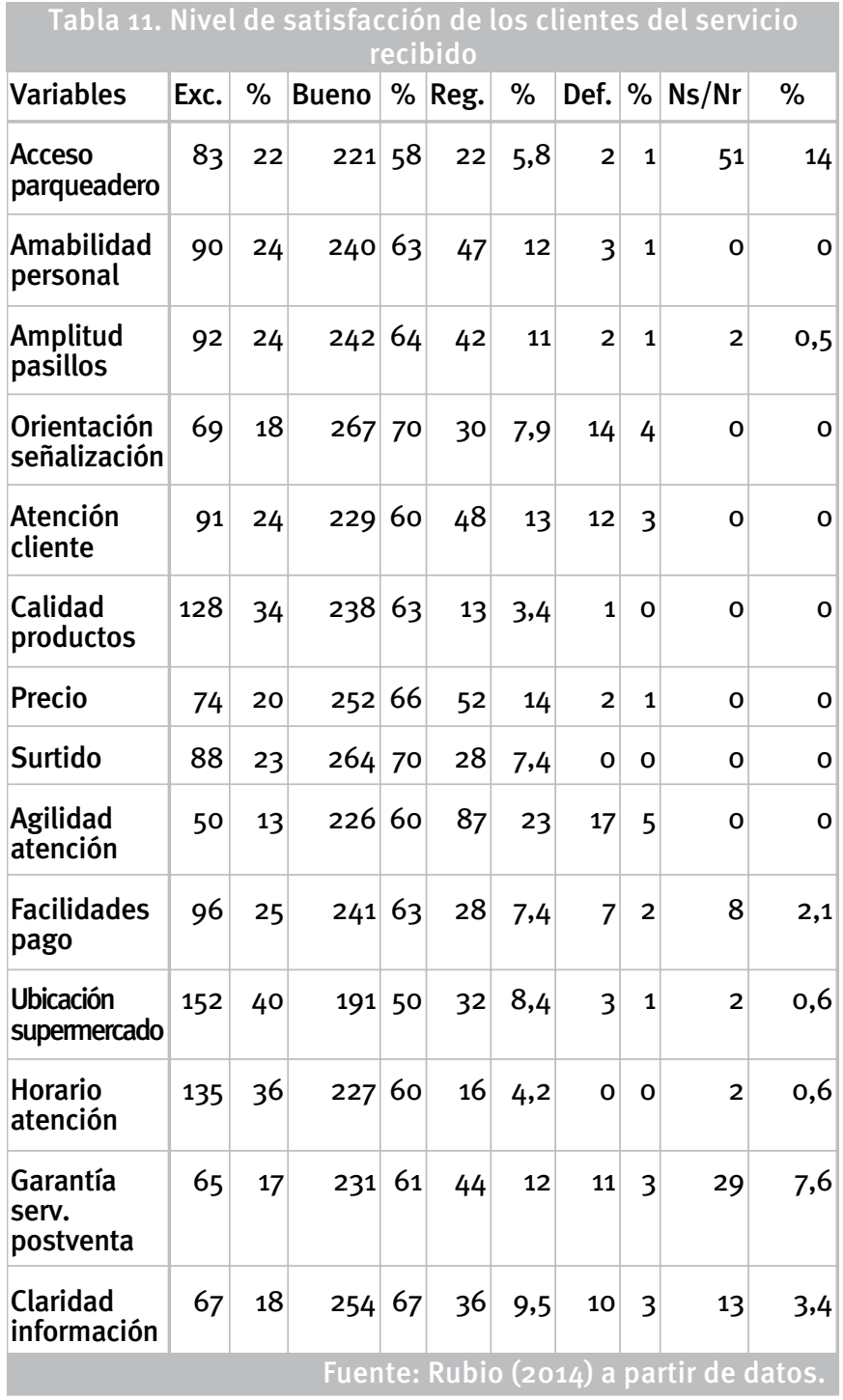

de empatía, los clientes valoran especialmente la ubicación del supermercado en razón a la cercanía desde sus viviendas, así como el horario de atención en que operan estas superficies. 
Las demás variables si bien es cierto no obtuvieron calificaciones representativas en la investigación, es importante no descuidarlas y por el contrario hacerles mayor énfasis o en su defecto mantenerlas, como una estrategia primordial en la conservación de la fidelidad de sus consumidores.

De otra parte también es importante considerar con base en Guisande et al. (2011), a instancias de las relaciones de asociación y dependencia de caracteres o variables (coeficiente de correlación de Pearson) y el análisis factorial por componentes principales como técnica de análisis confirmatorio, que los factores representados en las 5 dimensiones de la escala SERVQUAL de Parasuraman et. al. $(1985,1988)$, tienen una influencia relativa sobre la calidad percibida por los clientes que visitan estos supermercados, es decir, podría decirse que la percepción de la calidad de los clientes depende del constructo "calidad del servicio". En este mismo sentido se encontró que existe dependencia de la satisfacción del servicio con respecto a algunos de los aspectos tangibles considerados (parqueadero, calidad de los productos y surtido); igualmente la variable "claridad de la información de la oficina de servicio al cliente", predice el comportamiento del nivel de satisfacción del servicio. En esta misma dirección el nivel de satisfacción de los clientes depende de todas las variables de las dimensiones "seguridad" y "empatía".

Estudios posteriores podrían avanzar más en relaciones cruzadas de dependencia e independencia para el tratamiento de estas variables, en especial con la utilización de la técnica de ecuaciones estructurales, que comprende el manejo de correlaciones, covarianzas y relaciones causales entre variables latentes y observadas, a través de sus modelos de medida y estructura.

Por último es importante resaltar que existe una proporción importante de variables (atención al cliente, agilidad en la atención, garantía y servicio postventa, y amabilidad del personal entre otras), que no fueron bien calificadas por los clientes y que requieren un cuidado especial por parte de estas organizaciones.

\section{Referencias}

Aurifeille, J. M., Quester, P.G., Lockshin, L., \& Spawton, T. (2002), Global vs. international involvement-based segmentation: A cross-national exploratory study. International Marketing Review, 19 (4), 369-386

Bitner, M. J. y Hubbert, A. R. (1994). Encounter satisfaction versus overall satisfaction versus quality: the customer's voice. Service quality: new directions in theory and practice (pp. 72-94), Thousand Oaks, USA: Sage

Boulding, W., Kalra, A., Staelin, R., \& Zeithaml, V. (1993). A dynamic process model of service quality: from expectations to behavioral intentions. Journal of marketing research, (30), 7-27.

Crosby, P. (1979). Quality is free: The Art of Making Quality Certain. New York, USA: New American Library.
Cronbach, L. (1951). Coefficient alpha and the internal structure of tests. Psychometrika, 16 (3), 297-334.

Cronin, J., \& Taylor, S. (1992). Measuring service quality: a reexamination and extension. Journal of marketing, 56 (3), 55-68.

Dabholkar, P., Thorpe, D., \& Rentz, J. (1996). A measure of service quality for retail stores: scale development and validation. Journal Academy of marketing science, 24 (1), 3-16.

Deslauries, J. (2004). Investigación cualitativa: guía práctica. Pereira, Colombia: Editorial Papiro.

Ghauri, P., \& Gronhaug. K. (2010). Research Methods in Business Studies ( $4^{\mathrm{a}}$ ed.). Essex, England: Pearson.

Garvin, D. A. (1983). Quality on the Line. Harvard Business Review, 61, 65-73.

Gómez, M., Deslauries, J., y Alzate, M. (2010). Cómo hacer tesis de maestría y doctorado. Bogotá, Colombia: Ecoe Ediciones.

Guisande, C., Vaamonde, A., y Barreiro, A. (2011). Tratamiento de datos con R, Statistica y SPSS. Madrid, España: Díaz de Santos.

Hair, J.F., Anderson. R.E.,Tatham, R.L., y Black, W.C. (1999). Análisis Multivariante ( $5^{\underline{a}}$ ed pp. 79-81). Madrid, España: Prentice Hall.

Hayes, R., \& Wheelwright, S. (1984). Restoring our competitive edge: Competing through manufacturing. Nueva York, USA: Wiley.

Hernández, R., Fernández, C., y Baptista, P. (2010). Metodología de la investigación. ( $5^{\underline{a}}$ ed.). México D.F, México: Mc Graw Hill.

Holbrook, M.B. (1994). The nature of customer value: An axiology of services in the consumption experience. In Rust, $R$. T., \& Oliver, R. L., (Eds.). Service Quality-New Directions in Theory and Practice (pp. 21-71). Newbury Park, USA: Sage.

Huang, M. (2009). Using service quality to enhance the perceived quality of the store brands. Total Quality Management, 20 (2), 241-252.

Juran, J. (1988). Juran's quality control handbook. ( $4^{\mathrm{a}}$ ed.). New York, USA: McGraw Hill.

Kagira, E.K., \& Kimani, S.W. (2010). Marketing principles and practices: An African perspective. Nairobi, Africa: Mashel Publisher.

Kimani, S., Kagira, E., Kendi, L., \& Wawire, C. (2012). Shoppers perception of retail service quality: supermarkets versus small convenience shops (Dukas) in Kenya. Journal of management and strategy, 3 (1), 55-66.

Kotler, P. (2000). Marketing management: analysis, planning, implementation, and control. Upper Saddle River, USA: Prentice Hall.

Lewis, B.R. (1989). Quality in the service sector: A review. International Journal of Bank Marketing, 7 (5), 4-12.

Liu, K. (2007). Unfolding the post-transition era: the landscape and mindscape of China's retail industry after 2004. Asia Pacific Journal of Marketing and Logistics, 19 (4), 398-412.

Lind, D., Marchal, W., y Mason, R. (2004). Estadística para administración y economía. (11 $\mathrm{a}$ ed.). México, D. F, México: Alfa omega.

Martín-Peña, M.L., y Díaz-Garrido, E. (2009). Posicionamiento estratégico de las empresas industriales en las prioridades competitivas de operaciones: desarrollo y aplicación de un indicador de medida. Cuadernos de Economía y Dirección de la Empresa, (39). Recuperado de http://ehis.ebscohost. com/eds/pdfviewer/pdfviewer?vid=2\&sid=27b3c1fa-32a 8441d-aacc-e8ofo 245 c236\%40sessionmgr13\&hid $=8$ 
Martín, Q., Cabero, M.T., y De Paz, Y. (2008). Tratamiento estadístico de datos con SPSS prácticas resueltas y comentadas. Madrid, España: Thomson.

Méndez, C. (1995). Metodología: guía para elaborar diseños de investigación en ciencias económicas, contables y administrativas. Bogota, Colombia: Mc Graw Hill.

Meng, J., Summey, J., Herndon, N.C., \& Kwong, K. (2009). Some retail service quality expectations of Chinese shoppers. International Journal of Market Research, 51 (6), 773-796.

Miltenburg, J. (2009). Setting manufacturing strategy for a company's international manufacturing network. International Journal of Production Research, 47 (22). Recuperado de http://ehis.ebscohost.com/eds/pdfviewer/ pdfviewer?vid=2\&sid=decb9df8-deoo-4d43-8619-8defee bedb79\%40sessionmgr14\&hid=8

Nunally, J.C. (1978). Psychometric theory. New York, USA: McGraw Hill.

Olshavsky, R. (1985). Towards a More Comprehensive Theory of Choice. In NA - Advances in Consumer Research, 12, 465470.

Ospina, D. (2001). Introducción al muestreo. Bogotá, Colombia: Unibiblos Universidad Nacional de Colombia

Parasuraman, A., Zeithaml, V., \& Berry, L. (1985). A conceptual model of service quality and its implications for future research. Journal of Marketing, 49, 41-50.

Parasuraman, A., Zeithaml, V., \& Berry, L. (1988). SERVQUAL: A multiple-item scale for measuring consumer perceptions of service quality. Journal of retailing, 64 (1), 12-40.
Parasuraman, A., Zeithaml, V., \& Berry, L. (1991). Refinement and reassessment of the SERVQUAL scale. Journal of Retailing, 67 (4), 420-450.

Parasuraman, A., Zeithaml, V., \& Berry, L. (1994). Reassessment of expectations as a comparison standard in measuring service quality: implications for further research. Journal of Marketing, 58 (1), 111-124.

Radomir, L., Plaias, I., \& Nistor, V. (2012). A review of the service quality concept-past, present and perspectives. Marketing-from information to decision, 5, 404-427.

Santhiyavalli, G., \& Sandhya, B. (2011). Service quality evaluation in select commercial Banks: a comparative study. The IUP Journal of operations management, X, (1), 43-62.

Seth, N., Deshmukh, S., \& Vrat, P. (2004). Service quality models: a review. International Journal of Quality y Reliability Management, 22 (9), 913-949.

Sirohi, N., McLaughlin, E. W., \& Wittink, D. R. (1998). A model of consumer perceptions and store loyalty intentions for a supermarket retailer. Journal of Retailing, 74 (2), 223-245.

Siu, N. Y., \& Chow, D. K. (2003). Service quality in grocery retailing the study of a Japanese supermarket in Hong Kong. Journal of International Consumer Marketing, 16 (1), 71-87.

Skinner, W. (1969). Manufacturing-missing link in corporated strategy. Harvard Business Review, 47 (3), 136-145

Zeithaml, V. (1988). Consumer perceptions of price, quality and value: A means-end model and synthesis of evidence. Journal of Marketing, 52 (3), 2-22.

Cuadernos de Administración / Facultad de Ciencias de la Administración / Universidad del Valle

Periodicidad: semestral / ISSN impreso Nº120-4645 - ISSN electrónico Nº 2256-5078 / Nombre abreviado: cuad.adm.

Edición Vol. $31 \mathrm{~N}^{\circ} 52$ (julio - diciembre de 2014)

La calidad del servicio al cliente en los grandes supermercados de Ibagué: un análisis desde la escala multidimensional

(SERVQUAL) / Germán Rubio-Guerrero 